\title{
AEROMAGNETIC MAP OF THE MCNAIR-GRAND PORTAGE AREA NORTHEASTERN MINNESOTA
}

GEOPHYSICAL INVESTIGATIONS

MAP GP-639 